\title{
Citrin deficiency as a cause of chronic liver disorder mimicking nonalcoholic fatty liver disease
}

Michiharu Komatsu, ${ }^{1}$ Masahide Yazaki, ${ }^{2}$ Naoki Tanaka, ${ }^{1,3}$ Kenji Sano, ${ }^{4}$ Etsuko Hashimoto, ${ }^{5}$ Yo-ichi Takei, ${ }^{2}$ Yuan-Zong Song, ${ }^{6}$ Eiji Tanaka, ${ }^{1}$ Kendo Kiyosawa, Takeyori Saheki, ${ }^{8}$ Toshifumi Aoyama, ${ }^{3}$ and Keiko Kobayashi ${ }^{6}$

Departments of ${ }^{1}$ Gastroenterology and ${ }^{2}$ Neurology, Shinshu University School of Medicine, ${ }^{3}$ Department of Metabolic Regulation, Shinshu University Graduate School of Medicine, and ${ }^{4}$ Department of Laboratory Medicine, Shinshu University Hospital, Matsumoto, ${ }^{5}$ Institute of Gastroenterology, Tokyo Women's Medical University, Tokyo, ${ }^{6}$ Department of Molecular Metabolism and Biochemical Genetics, Kagoshima University Graduate School of Medical and Dental Sciences, Kagoshima, ${ }^{7}$ Department of Internal Medicine, Nagano Red Cross Hospital, Nagano, and ${ }^{8}$ Institute for Health Sciences, Tokushima Bunri University, Tokushima, Japan

Corresponding author: Naoki Tanaka, MD, PhD

Department of Metabolic Regulation, Shinshu University Graduate School of Medicine, Asahi 3-1-1, Matsumoto, 390-8621, Japan

Fax: +81-263-37-3094

e-mail: naopi@hsp.md.shinshu-u.ac.jp 
Running title: Fatty liver disease associated with citrin deficiency

Conflict of interests: none

Word counts: 3421

Abbreviations: AGC, aspartate-glutamate carrier; NADH, nicotinamide adenine dinucleotide; ATP, adenosine triphosphate; ASS, argininosuccinate synthetase; NICCD, neonatal intrahepatic cholestasis caused by citrin deficiency; CTLN2, adult-onset type II citrullinemia; NAFLD, nonalcoholic fatty liver disease; NASH, nonalcoholic steatohepatitis; US, ultrasonography; CT, computed tomography; HCV, hepatitis C virus; ALT, alanine aminotransferase; BMI, body mass index; HOMA-IR, homeostasis model assessment of insulin resistance; PSTI, pancreatic secretory trypsin inhibitor; NAS, NAFLD activity score; ROC, receiver-operating characteristic; AUC, area under the ROC curve; $\gamma \mathrm{GT}$, $\gamma$-glutamyltransferase; HCC, hepatocellular carcinoma. 


\begin{abstract}
Background/Aims: Citrin deficiency caused by SLC25A13 gene mutations develops into adult-onset type II citrullinemia (CTLN2) and may be accompanied with hepatic steatosis and steatohepatitis. As its clinical features remain unclear, we aimed to explore the characteristics of fatty liver disease associated with citrin deficiency.

Methods: The prevalence of hepatic steatosis in 19 CTLN2 patients was examined, and clinical features were compared with those of nonalcoholic fatty liver disease (NAFLD) patients without known SLC25A13 gene mutations.

Results: Seventeen (89\%) CTLN2 patients had steatosis, and $4(21 \%)$ had been diagnosed as having NAFLD before appearance of neuropsychological symptoms. One patient had steatohepatitis. Citrin deficiency-associated fatty livers showed a considerably lower prevalence of accompanying obesity and metabolic syndrome, higher prevalence of history of pancreatitis, and higher serum levels of pancreatic secretory trypsin inhibitor (PSTI) than fatty livers without the mutations. Receiver operating characteristic curve analyses revealed that a body mass index $<20 \mathrm{~kg} / \mathrm{m}^{2}$ and serum PSTI $>29 \mathrm{ng} / \mathrm{mL}$ were associated with citrin deficiency.

Conclusion: Patients presenting with nonalcoholic fatty liver unrelated to obesity and metabolic syndrome might have citrin deficiency, and serum PSTI may be a useful indicator for distinguishing this from conventional NAFLD.
\end{abstract}




\section{Introduction}

Citrin is a liver-type mitochondrial aspartate-glutamate carrier (AGC) known to exchange mitochondrial aspartate for cytosolic glutamate and a proton [1-3]. This function is important in translocating cytosolic nicotinamide adenine dinucleotide $(\mathrm{NADH})$ reducing equivalents into the mitochondria as a part of the malate-aspartate shuttle, and NADH produced by malate is oxidized to generate adenosine triphosphate (ATP) in the oxidative phosphorylation pathway. Moreover, liver-specific AGC also plays an important role in supplying aspartate to argininosuccinate synthetase (ASS) in the cytosol to generate argininosuccinate in the urea cycle. Thus, a deficiency of liver-specific AGC, namely citrin, results in dysfunction of the urea cycle and hyperammonemia $[2,3]$.

Citrin deficiency is an autosomal recessive disorder caused by a mutation of the SLC25A13 gene encoding citrin, which is located on chromosome 7q21.3 [2-4]. Some of the mutations identified in Japanese patients are frequently found in the East Asian population, and the prevalence of these mutations has been estimated to be $1 / 65$ in China, 1/112 in Korea, and 1/69 in Japan [3, 5, 6]. Furthermore, several cases with SLC25A13 gene mutations have been reported in Israel, USA, and elsewhere [7-9], indicating a world-wide incidence of citrin deficiency.

The SLC25A13 gene mutation leads to neonatal intrahepatic cholestasis caused by citrin deficiency (NICCD) and adult-onset type II citrullinemia (CTLN2) [2, 3]. Citrin deficiency is sometimes asymptomatic in adults probably by metabolic adaptation, but CTLN2 is characterized by the sudden onset of various neuropsychological symptoms including disorientation, abnormal behavior, convulsions, and coma due to hyperammonemia, which may result in rapidly progressive and irreversible brain edema and death. Generally speaking, the prognosis of patients with CTLN2 is not favorable after the onset of encephalopathy, and liver transplantation is required for severe CTLN2 which is refractory to various ammonia-lowering and neuroprotective therapies. Furthermore, certain types of conventional treatments for brain edema, such as intravenous administration of hyperosmotic and high sugar solutions, have been reported to aggravate encephalopathy $[2,10,11]$, and the mechanism of toxicity of carbohydrate overload for citrin deficiency has been recently clarified using mouse models [12]. Thus, early diagnosis of citrin deficiency and CTLN2 and appropriate treatment might improve prognosis.

Citrin deficiency has been demonstrated to present with hepatic steatosis and steatohepatitis. Previously, we reported the case of a patient with nonalcoholic fatty liver disease (NAFLD) who was later shown to carry SLC25A13 gene mutation [13]. The patient had elevation of serum aminotransferase levels and fatty liver of unknown causes, and was first diagnosed as having NAFLD by liver biopsy at the age of 23 . Ten years afterwards, serious encephalopathy suddenly appeared after accidental consumption of alcohol. The patient was then diagnosed as having CTLN2, but did not respond to any conservative treatments and required urgent liver transplantation $[13,14]$. Moreover, Takagi et al. have also reported three CTLN2 patients with liver histologies of steatohepatitis [15]. These findings suggest the possibility that citrin deficiency may be present in adults who are diagnosed as having NAFLD or nonalcoholic steatohepatitis (NASH), and elucidating the characteristics of fatty liver related to citrin deficiency may be helpful in the early distinction of this disease from conventional NAFLD. However, the clinical features of this phenomenon have not been fully 
investigated.

In the present study, we evaluated the clinical characteristics of citrin deficiency-associated fatty liver through analysis of CTLN2 patients presenting with fatty liver, and sought to find key indicators to detect citrin deficiency in NAFLD patients. 


\section{Methods}

\subsection{Patients}

Nineteen CTLN2 patients (11 men and 8 women, mean age $37+10 \mathrm{yrs}$ ), who had been admitted to Shinshu University Hospital between 1979 and 2007, were examined in this study. All patients had experienced neuropsychological symptoms due to hyperammonemia. CTLN2 was suspected from the presence of neurological abnormalities and elevated plasma ammonia and citrulline levels, and diagnosis was confirmed by the presence of SLC25A13 gene mutations. In these patients, the presence of fatty liver was confirmed by liver histology (i.e., lipid accumulation in more than 5\% of hepatocytes) or imaging modalities such as ultrasonography (US) and/or computed tomography (CT) scan.

For comparison, 25 NAFLD patients (10 men and 15 women, mean age $61+13$ yrs), who had been admitted to Shinshu University Hospital between 2003 and 2004 for the purpose of percutaneous liver biopsy, were analyzed. These patients did not have any of the 16 different mutations frequently found in the SLC25A13 gene of Japanese patients with citrin deficiency. Three patients were diagnosed as having NASH according to the histological criteria proposed by Kleiner et al [16].

NAFLD patients without SLC25A13 mutations satisfied the following exclusion criteria: no consumption of alcohol or steatosis-inducing drugs such as tamoxifen or anti-retroviral agents, negative results for hepatitis B surface antigen, anti-hepatitis B core and anti-hepatitis $\mathrm{C}$ virus (HCV) antibodies, and no presence of other types of chronic liver disease such as autoimmune liver diseases, Wilson's disease, hereditary hemochromatosis or $\alpha 1$-antitrypsin deficiency.

\subsection{Analysis of clinical features}

At the time of admission, body height, weight, and waist circumference were measured by hospital staff unaware of the patients' medical information, and clinical course and past history were taken for each patient and family. History of alcohol or drug intake and fondness for foods were also recorded. The time of diagnosis of CTLN2 was taken as the age of appearance of neuropsychological symptoms and detection of hyperammonemia and/or hypercitrullinemia. The time of diagnosis of fatty liver was taken as the age of diagnosis by liver biopsy or imaging. The time of diagnosis of hyperlipidemia, type 2 diabetes, and elevation of alanine aminotransferase (ALT) level (>35 U/L) was taken as the age of detection of these abnormalities by blood examination. The time of diagnosis of pancreatitis was taken as the age of diagnosis by physical, laboratory, and imaging examinations.

The presence of obesity was defined as having a body mass index (BMI) of more than $25 \mathrm{~kg} / \mathrm{m}^{2}$, based on criteria released by Japan Society for the Study of Obesity [17]. Patients were considered to be hypertensive if their systolic/diastolic blood pressure was greater than $140 / 90 \mathrm{mmHg}$, or if they were taking anti-hypertensive drugs. Patients were considered to be diabetic if they had a fasting glucose level equal to or higher than $7.0 \mathrm{mmol} / \mathrm{L}(126 \mathrm{mg} / \mathrm{dL})$ or if they were taking insulin or oral hypoglycemic drugs. Patients were considered to have hyperlipidemia if their fasting serum levels of cholesterol or triglyceride were equal to or higher than $5.7 \mathrm{mmol} / \mathrm{L}(220 \mathrm{mg} / \mathrm{dL})$ or 1.7 $\mathrm{mmol} / \mathrm{L}(150 \mathrm{mg} / \mathrm{dL})$, respectively, or if they were taking lipid-lowering drugs [18]. The presence of metabolic syndrome was judged according to the criteria released by the Japanese Committee for the Diagnostic Criteria of Metabolic Syndrome [19]. 


\subsection{Laboratory and biochemical examination}

At the time of admission, blood samples were obtained in a fasting state, and complete blood counts and blood chemistries, including lipoprotein, were determined by standard methods. The homeostasis model assessment of insulin resistance (HOMA-IR), a simple and reliable indicator of insulin resistance, was calculated by the following formula: [fasting plasma glucose $(\mathrm{mmol} / \mathrm{L}) \mathrm{x}$ immunoreactive insulin $(\mu \mathrm{U} / \mathrm{mL})] / 22.5$. A HOMA-IR of greater than 2 was defined as the presence of insulin resistance [18]. Serum pancreatic secretory trypsin inhibitor (PSTI) concentration was measured by immunoradiometric assay (Eiken Chemical Co., Ltd, Tokyo, Japan). Hepatic ASS activity was measured as described by $\mathrm{Su}$ et al [20], and values were expressed as percentages of control.

\subsection{Imaging examination}

Each patient underwent abdominal US or CT scan. The presence of fatty liver was assessed according to positive findings such as hepatorenal contrast, blurring of the vascular wall, and/or profound attenuation of the diaphragm $[18,21]$. In CT scans, the presence of hepatic steatosis was assessed according to criteria described elsewhere $[22]$.

\subsection{Liver histology}

Liver samples were obtained from 14 CTLN2 patients with fatty liver by percutaneous US-guided biopsy (6 patients; Nos. 1, 7, 8, 11, 12, and 14 in Table 1) or from explanted livers at living donor liver transplantation (8 patients). Liver samples were fixed in $10 \%$ neutral formalin, and sections cut at $4-\mu \mathrm{m}$ thickness were stained with hematoxylin and eosin or Azan-Mallory methods. Histological diagnosis was performed by two experienced pathologists $(\mathrm{KS}, \mathrm{EH})$ in a blinded fashion. The NAFLD activity score (NAS) was assessed according to the criteria proposed by Kleiner et al [16]. The diagnosis of NASH was confirmed by a NAS of more than 5 .

\subsection{SLC25A13 gene mutation analysis}

DNA was extracted from peripheral blood using standard methods. Sixteen SLC25A13 mutations found relatively frequently in Japan, 13 mutations described previously [4, 6, 23-25] and three unpublished novel mutations (Tabata et al., manuscript in preparation), were tested by the polymerase chain reaction-restriction fragment length polymorphism method and/or multiple methods using Genescan/SNaPshot. A novel mutation identified by PCR/sequencing of all 18 exons and the flanking sequences in an allele of a CTLN2 patient (No. 8 in Table 1) will be described elsewhere (Song et al., manuscript in preparation).

\subsection{Ethics}

This study was approved by the ethical committees of the Shinshu University School of Medicine and Kagoshima University Faculty of Medicine, and adheres to the principles of the Declaration of Helsinki.

\subsection{Statistics}

Categorical variables were expressed as percentages, and continuous variables 
were expressed as median and range (in parentheses). Statistical analyses were performed using SPSS software 11.5J for Windows (SPSS Inc., Chicago, IL). Comparisons were made using Fisher's exact probability test for categorical variables, the $\chi^{2}$ test for histological scores, and the Mann-Whitney $U$-test for continuous variables. To assess the clinical parameters in differentiating citrin deficiency from NAFLD, receiver-operating characteristic (ROC) curves were constructed by plotting sensitivity against reverse specificity ( 1 minus specificity) for each value. In this assessment, a larger area under the ROC curve (AUC) corresponded to a more useful marker for differentiating citrin deficiency. The most appropriate cut-off point was the point at which the sum of the sensitivity and specificity was maximized. A probability value of less than 0.05 was considered to be statistically significant. 


\section{Results}

\subsection{Prevalence of fatty liver in CTLN2 patients at time of admission}

Clinical features of the 19 CTLN2 patients are summarized in Table 1. Their median age was 40 years. Only one patient (No. 11) had a past history of prolonged neonatal jaundice, a symptom suggestive of NICCD [26]. Thirteen (68\%) patients were fond of eating protein-rich foods such as beans, eggs, or cheese, but the others did not have any peculiar dietary preferences. All patients demonstrated hypercitrullinemia and had SLC25A13 gene mutations (9 homozygotes), and all patients except one (No. 9) demonstrated marked reduction in hepatic ASS activity. Elevation of serum ALT and $\gamma$-glutamyltransferase $(\gamma \mathrm{GT})$ levels and fatty liver were observed in 17 (89\%), 15 (79\%), and $17(89 \%)$ patients, respectively, at the time of admission (Table 2). None of the patients had a history of alcohol drinking. Interestingly, in CTLN2 patients with fatty liver, median BMI values were as low as $18.3 \mathrm{~kg} / \mathrm{m}^{2}$, and none was classified as obese (Table 2). Increases in waist circumference, a reliable anthropometric indicator of visceral obesity, were also not observed in these patients. These results corroborate with the high prevalence of non-obese nonalcoholic fatty liver in CTLN2 patients.

\subsection{Time of diagnosis}

Next, the times of diagnosis of CTLN2, hyperlipidemia, fatty liver, elevation of serum ALT levels, and pancreatitis were recorded (Table 3). The median age of the initial diagnosis of CTLN2 was 40 years. Hypertriglyceridemia was present in $6(32 \%)$ patients. Interestingly, hypertriglyceridemia, fatty liver, and elevation of serum ALT levels had been pointed out in $3(16 \%), 5(26 \%)$, and $8(42 \%)$ patients, respectively, before appearance of neuropsychological symptoms, i.e., diagnosis of CTLN2. Furthermore, $5(26 \%)$ patients had experienced pancreatitis, all of which preceded diagnosis with CTLN2.

\subsection{Histological findings of fatty liver with citrin deficiency}

In the 17 CTLN2 patients with fatty liver, one patient (No. 17) was excluded because of positive results for anti-HCV antibody and serum HCV-RNA (HCV genotype 1b). The remaining 16 patients (Nos. 1-16) had hepatic steatosis, and liver samples were obtained from 14 patients (Nos. 1-14) (Table 4). All samples demonstrated various degrees of macrovesicular steatosis mainly present in zone 3 . Apparent microvesicular steatosis was also present in $4(29 \%)$ samples. Perisinusoidal fibrosis was detected in $11(79 \%)$ samples, and one (8\%) sample demonstrated bridging fibrosis (Figure 1A). Mild lobular and portal inflammation consisting of mononuclear cell infiltration was observed in $12(86 \%)$ and $6(43 \%)$ samples, respectively. Hepatocyte ballooning, Mallory hyaline, and glycogenated nuclei were detected in 4 $(29 \%), 1(7 \%)$, and $1(7 \%)$ sample, respectively. Assessment of histological severity of NAFLD revealed that 1 (7\%) patient (No. 6) had a NAS of more than 5 (Figure 1B), but approximately $50 \%$ of the patients had a NAS of less than 3 .

3.4. Comparison of clinical and biochemical findings between fatty liver with and without SLC25A13 gene mutations

In order to investigate the clinical characteristics of fatty liver associated with citrin deficiency, clinical and biochemical findings of these patients were compared with fatty liver cases without SLC25A13 mutations, i.e., conventional NAFLD. As shown in Table 
5, fatty liver patients with the mutations had a lower frequency of obesity ( 0 vs $72 \%, P$ $<0.001)$, metabolic syndrome ( 0 vs $36 \%, P<0.001)$, type 2 diabetes $(0$ vs $32 \%, P<$ 0.001 ), hypertension ( 0 vs $44 \%, P<0.001$ ), and a higher frequency of history of pancreatitis (13 vs $0 \%, P=0.002)$ than those without the mutations. The median BMI of citrin deficiency patients was significantly lower $\left(18.3\right.$ vs $27.3 \mathrm{~kg} / \mathrm{m}^{2}, P<0.001$ ), as were waist circumference ( 72 vs $89 \mathrm{~cm}, P<0.001)$, serum albumin $(3.7$ vs $4.4 \mathrm{~g} / \mathrm{dL}, P$ $=0.001)$, cholesterol $(4.8$ vs $5.8 \mathrm{mmol} / \mathrm{L}, P=0.003)$, low-density-lipoprotein cholesterol (2.1 vs $4.2 \mathrm{mmol} / \mathrm{L}, P=0.002)$, and HOMA-IR (1.7 vs $4.8, P=0.006)$ (Table 5). Serum PSTI concentrations were significantly greater in patients with citrin deficiency (74 vs $10 \mathrm{ng} / \mathrm{mL}, P<0.001$ ).

\subsection{ROC curve analysis}

We first tried multivariate logistic regression analysis to identify key clinical parameters for detecting citrin deficiency in NAFLD, but were unable to obtain any meaningful data due to the remarkable differences in BMI and PSTI. Thus, ROC curves were constructed and evaluated for each parameter. The AUC of BMI and PSTI were found to be quite large ( 0.968 and 0.989 , respectively). The odds ratio for BMI was 0.48 (95\% confidence interval, 0.924-1.011; $P<0.001)$, and that for PSTI was $1.37(95 \%$ confidence interval, 0.963-1.015; $P<0.001)$. 


\section{Discussion}

The present study demonstrates that citrin deficiency can superficially mimic NAFLD before the onset of neurological abnormalities. However, fatty liver patients with SLC25A13 mutations lacked the complications of obesity, metabolic syndrome, and diabetes. Furthermore, BMI and serum PSTI appeared to be clues in distinguishing citrin deficiency from conventional NAFLD. As far as we know, this is the first study to review the clinical features of citrin deficiency-associated fatty liver disease and compare the characteristics with those of NAFLD without SLC25A13 mutations.

As evidenced in Table 3, it is plausible that many patients homozygous for SLC25A13 mutations may be diagnosed as having other diseases, such as NAFLD, hyperlipidemia, and pancreatitis, before the appearance of neurological abnormalities and diagnosis of CTLN2. There is also great variety in the time and mode of onset of neurological episodes in CTLN2; some patients do not experience neurological symptoms until middle or old age. For example, in one patient, the first neurological episode occurred at 79 years of age [23]. Indeed, the patient (No. 9), who had been diagnosed as having hepatocellular carcinoma (HCC) of unknown etiology experienced consciousness disturbance for the first time after hepatic resection at 51 years of age, leading to the diagnosis of CTLN2 [27]. This diversity may be partially explained by varying degrees of reduction in hepatic ASS activity. The frequency of homozygotes in Japan $(1 / 19,000)$ [3] calculated from carrier rate is quite different from the actually reported incidence of CTLN2 $(1 / 100,000)$ [28], suggesting that many people with citrin deficiency devoid of neurological symptoms may exist in the general population.

The mechanism of steatogenesis by citrin deficiency may be explained as follows: $\mathrm{NADH}$ reducing equivalents are supplied to the mitochondria mainly via the malate-aspartate shuttle in human hepatocytes (Figure 3A). However, citrin deficiency loses this shuttle, resulting in the compensatory up-regulation of the malate-citrate shuttle, which increases citrate in the cytosol (Figure 3B). A large amount of citrate is converted to acetyl-CoA by ATP-citrate lyase, further leading to overproduction of fatty acids in hepatocytes (Figure 3C). In fact, elevated mRNA levels of ATP-citrate lyase have been confirmed in livers of citrin/mitochondrial glycerol-3-phosphate dehydrogenase double knockout mouse, a suitable model of human citrin deficiency [12]. The mRNA levels of CD36 (fatty acid translocase) were also elevated in these mouse livers [12], suggesting increased fatty acid uptake capacity into hepatocytes. Based on these findings, enhancement of de novo lipogenesis and fatty acid uptake into hepatocytes are considered to be key events in the development of hepatic steatosis in patients with citrin deficiency. Additionally, the observation that plasma ketone body levels are relatively low in CTLN2 patients [29] implies that mitochondrial fatty acid-degrading capacity might be reduced to some degree [30, 31].

A striking finding in this study is that some citrin deficiency patients presenting with fatty liver had a history of pancreatitis, indicating a possible susceptibility to citrin deficiency in NAFLD/NASH patients who have experienced this disease. Pancreatitis unrelated to alcohol consumption, which is sometimes chronic and recurrent, is one of the hallmark features of citrin deficiency [32], but its etiology remains unclear. Recently, we reported the case of a non-obese patient with NASH caused by pancreatic exocrine insufficiency [33]. This case was characterized by the absence of obesity and insulin resistance and decrease in serum levels of albumin and cholesterol, showing several resemblances to fatty liver with citrin deficiency. A significant reduction in plasma 
methionine concentrations was also observed in this case, and both hepatic steatosis and decreased plasma methionine levels were significantly improved by pancreatic enzyme supplementation [33]. Therefore, impaired pancreatic exocrine function due to chronic pancreatitis, latent malabsorption of essential amino acids, and the resultant deficiency of methionine might at least in part contribute to the pathogenesis of fatty liver with citrin deficiency.

The most remarkable characteristics of fatty liver disease due to citrin deficiency were low BMI and high serum PSTI. All CTLN2 patients with fatty liver were lean, which is consistent with other previous reports $[13,14,34,35]$. This phenomenon may derive from a unique food habit of disliking high calorie and high carbohydrate foods such as rice and sweets [2, 3]. An increase in serum PSTI is known to sometimes precede neurological episodes [36, 37]. Indeed, in a patient we reported [13], a retrospective measurement revealed a marked elevation of serum PSTI $(170 \mathrm{ng} / \mathrm{mL})$ at the time of liver biopsy in which he appeared conscious. Therefore, we may postulate that measurement of BMI and serum PSTI may be useful as an initial screening method to distinguish citrin deficiency from NAFLD. We are prospectively examining serum PSTI in non-obese NAFLD patients, but all patients analyzed thus far have had normal PSTI and no SLC25A13 gene mutations. Verifying this finding will require further large-scale examinations.

It is quite noteworthy that some CTLN2 patients with steatosis developed severe fibrosis (Figure 1A) or cirrhosis, suggesting that citrin deficiency may progress to cirrhosis. Since all liver samples analyzed were obtained shortly after the onset of neurological abnormalities and the initiation of ammonia-reducing therapy, the possibility that the development of hepatic fibrosis can be attributed to inadequate therapeutic interventions (e.g., strict protein restriction, long-term intravenous hyperalimentation) seems very low. More importantly, citrin deficiency can induce HCC $[2,27,38-40]$, meaning clinicians should also consider citrin deficiency as one of the etiologies of cryptogenic cirrhosis or HCC.

The results found in the current study lead to several points that need further study. Firstly, the prevalence of citrin deficiency in patients with cryptogenic chronic liver diseases should be clarified. Secondly, simple, non-invasive, and useful indicators to find apparently healthy homozygotes need to be established. For instance, the usefulness of immunoblot analysis of citrin by means of lymphocytes has recently been documented [41]. Finally, possible pharmacological interventions against asymptomatic citrin deficiency patients should be discussed as therapeutic interventions to decrease cytosolic citrate might attenuate hepatic steatosis. A recent study using citrin-deficient mice demonstrated that pyruvate replenishment may be effective to alleviate metabolic disturbances [42].

In conclusion, patients who present with fatty liver, especially non-obese individuals, might have citrin deficiency mimicking NAFLD. Medical history, dietary preference, and anthropometric and laboratory data such as BMI and serum PSTI may provide clues to discriminate citrin deficiency from NAFLD. 


\section{Acknowledgments}

The authors would like to thank Mr. Trevor Ralph for his editorial assistance. This study was supported in part by Grant-in-Aids for Scientific Research (B: No. 16390100 and 19390096) and for the Asia-Africa Scientific Platform Program from the Japan Society for the Promotion of Science. 


\section{References}

[1] Palmieri L, Pardo B, Lasorsa FM, del Arco A, Kobayashi K, Iijima M, et al. Citrin and aralar1 are $\mathrm{Ca}^{2+}$-stimulated aspartate/glutamate transporters in mitochondria. EMBO J 2001; 20: 5060-5069.

[2] Saheki T, Kobayashi K, Iijima M, Moriyama M, Yazaki M, Takei Y, et al. Metabolic derangements in deficiency of citrin, a liver-type mitochondrial aspartate-glutamate carrier. Hepatol Res 2005; 33: 181-184.

[3] Saheki T, Kobayashi K. Mitochondrial aspartate glutamate carrier (citrin) deficiency as the cause of adult-onset type II citrullinemia (CTLN2) and idiopathic neonatal hepatitis. J Hum Genet 2002; 47: 333-341.

[4] Kobayashi K, Sinasac DS, Iijima M, Boright AP, Begum L, Lee JR, et al. The gene mutated in adult-onset type II citrullinemia encodes a putative mitochondrial carrier protein. Nat Genet 1999; 22: 159-163.

[5] Kobayashi K, Lu YB, Li MX, Nishi I, Hisao KJ, Choeh K, et al. Screening of nine SLC25A13 mutations: their frequency in patients with citrin deficiency and high carrier rates in Asian populations. Mol Genet Metab 2003; 80: 356-359.

[6] Lu YB, Kobayashi K, Ushiki M, Tabata A, Iijima M, Li MX, et al. Frequency and distribution in East Asia of 12 mutations identified in the SLC25A13 gene of Japanese patients with citrin deficiency. J Hum Genet 2005; 50: 338-346.

[7] Ben-Shalom E, Kobayashi K, Shaag A, Yasuda T, Gao HZ, Saheki T, et al. Infantile citrullinemia caused by citrin deficiency with increased dibasic amino acids. Mol Genet Metab 2002; 77: 202-208.

[8] Hutchin T, Preece MA, Kobayashi K, Saheki T, Brown R, Kelly DA, et al. Neonatal intrahepatic cholestasis caused by citrin deficiency (NICCD) in a European patient. J Inherit Metab Dis 2006; 29 (Suppl. 1): 112.

[9] Dimmock D, Kobayashi K, Iijima M, Tabata A, Wong LJ, Saheki T, et al. Citrin deficiency: a novel cause of failure to thrive that responds to a high-protein, low-carbohydrate diet. Pediatrics 2007; 119: 773-777.

[10] Yazaki M, Takei Y, Kobayashi K, Saheki T, Ikeda S. Risk of worsened encephalopathy after intravenous glycerol therapy in patients with adult-onset type II citrullinemia (CTLN2). Intern Med 2005; 44: 188-195.

[11] Takahashi H, Kagawa T, Kobayashi K, Hirabayashi H, Yui M, Begum L, et al. A case of adult-onset type II citrullinemia: Deterioration of clinical course after infusion of hyperosmotic and high sugar solutions. Med Sci Monit 2006; 12: CS13-15.

[12] Saheki T, Iijima M, Li MX, Kobayashi K, Horiuchi M, Ushikai M, et al. Citrin/mitochondrial glycerol 3-phosphate double-knockout mice recapitulate features of human citrin deficiency. J Biol Chem 2007; 282: 25041-25052.

[13] Tanaka N, Yazaki M, Kobayashi K. A lean man with nonalcoholic fatty liver disease. Clin Gastroenterol Hepatol 2007; 5: A32.

[14] Yazaki M, Hashikura Y, Takei Y, Ikegami T, Miyagawa S, Yamamoto K, et al. Feasibility of auxiliary partial orthotopic liver transplantation from living donors for patients with adult-onset type II citrullinemia. Liver Transpl 2004; 10: 550-554.

[15] Takagi H, Hagiwara S, Hashizume H, Kanda D, Sato K, Sohara N, et al. Adult onset type II citrullinemia as a cause of non-alcoholic steatohepatitis. J Hepatol 2006; 44: 236-239. 
[16] Kleiner DE, Brunt EM, Natta MV, Behling C, Contos MJ, Cummings OW, et al. Design and validation of histological scoring system for nonalcoholic fatty liver disease. Hepatology 2005; 41: 1313-1321.

[17] The Examination Committee of Criteria for 'Obesity Diseases' in Japan, Japan Society for the Study of Obesity. New Criteria for 'Obesity Disease' in Japan. Circ J 2002; 66: 987-992.

[18] Tanaka N, Tanaka E, Sheena Y, Komatsu M, Okiyama W, Misawa N, et al. Useful parameters for distinguishing nonalcoholic steatohepatitis with mild steatosis from cryptogenic chronic hepatitis in the Japanese population. Liver Int 2006; 26: 956-963.

[19] Arai H, Yamamoto A, Matsuzawa Y, Saito Y, Yamada N, Oikawa S, et al. Prevalence of metabolic syndrome in the general Japanese population in 2000. J Atheroscler Thromb 2006; 13: 202-208.

[20] $\mathrm{Su}$ TS, Bock HG, Beaudet AL, O'Brien WE. Molecular analysis of argininosuccinate synthatase deficiency in human fibroblasts. J Clin Invest 1982; 70: 1334-1339.

[21] Tanaka N, Sano K, Horiuchi A, Tanaka E, Kiyosawa K, Aoyama T. Highly-purified eicosapentaenoic acid treatment improves nonalcoholic steatohepatitis. J Clin Gastroenterol, 2008; 42: 413-418.

[22] Saadeh S, Younossi ZM, Remer EM, Gramlich T, Ong JP, Hurley M, et al. The utility of radiological imaging in nonalcoholic fatty liver disease. Gastroenterology 2002; 123: 745-750.

[23] Yasuda T, Yamaguchi N, Kobayashi K, Nishi I, Horinouchi H, Jalil MA, et al. Identification of two novel mutations in the SLC25A13 gene and detection of seven mutations in 102 patients with adult-onset type II citrullinemia. Hum Genet 2000; 107: 537-545.

[24] Yamaguchi N, Kobayashi K, Yasuda T, Nishi I, Iijima M, Nakagawa M, et al. Screening of SLC25A13 mutations in early and late onset patients with citrin deficiency and in the Japanese population: identification of two novel mutations and establishment of multiple DNA diagnosis methods for nine mutations. Hum Mutat 2002; 19: 122-130.

[25] Takaya J, Kobayashi K, Ohashi A, Ushikai M, Tabata A, Fujimoto S, et al. Variant clinical courses of 2 patients with neonatal intrahepatic cholestasis who have a novel mutation of SLC25A13. Metabolism 2005; 54: 1615-1619.

[26] Ohura T, Kobayashi K, Tazawa Y, Abukawa D, Sakamoto O, Tsuchiya S, et al. Clinical pictures of 75 patients with neonatal intrahepatic cholestasis caused by citrin deficiency (NICCD). J Inherit Metab Dis 2007; 30: 139-144.

[27] Soeda J, Yazaki M, Nakata T, Miwa S, Ikeda S, Hosoda W, et al. Primary liver carcinoma exhibiting dual hepatocellular-biliary epithelial differentiations associated with citrin deficiency: a case report and review of the literature. J Clin Gastroenterol, in press.

[28] Kobayashi K, Shaheen N, Kumashiro R, Tanikawa K, O’Brien WE, Beaudet AL, et al. A search for primary abnormality in adult-onset type II citrullinemia. Am J Hum Genet 1993; 53: 1024-1030.

[29] Inui Y, Kuwajima M, Kawata S, Fukuda K, Maeda Y, Igura T, et al. Impaired ketogenesis in patients with adult-type citrullinemia. Gastroenterology 1994; 107 : 1154-1161. 
[30] Aoyama T, Peters JM., Iritani N, Nakajima T, Furihata K, Hashimoto T, et al. Altered constitutive expression of fatty acid-metabolizing enzymes in mice lacking the peroxisome proliferator-activated receptor $\alpha(\operatorname{PPAR} \alpha)$. J Biol Chem 1998; 273 : 5678-5684.

[31] Tanaka N, Moriya K, Kiyosawa K, Koike K, Gonzalez FJ, Aoyama T. PPAR $\alpha$ activation is essential for HCV core protein-induced hepatic steatosis and hepatocellular carcinoma in mice. J Clin Invest 2008, 118: 683-694.

[32] Ikeda S, Kawa S, Takei Y, Yamamoto K, Shimojo H, Tabata K, et al. Chronic pancreatitis associated with adult-onset type II citrullinemia: clinical and pathologic findings. Ann Intern Med 2004; 141: W109-110.

[33] Tanaka N, Horiuchi A, Yokoyama T, Kawa S, Kiyosawa K. Pancreatic exocrine insufficiency: a rare cause of nonalcoholic steatohepatitis. Am J Gastroenterol 2008; 103: 245-246.

[34] Ikeda S, Yazaki M, Takei Y, Ikegami Y, Hashikura Y, Kawasaki S, et al. Type II (adult onset) citrullinaemia: clinical pictures and the therapeutic effect of liver transplantation. J Neurol Neurosurg Psychiatry 2001; 71: 663-670.

[35] Imamura Y, Kobayashi K, Shibatou T, Aburada S, Tahara K, Kubozono O, et al. Effectiveness of carbohydrate-restricted diet and arginine granules therapy for adult-onset type II citrullinemia: a case report of siblings showing homozygous SLC25A13 mutation with and without the disease. Hepatol Res 2003; 26: 68-72.

[36] Kobayashi K, Horiuchi M, Saheki T. Pancreatic secretory trypsin inhibitor as a diagnostic marker for adult-onset type II citrullinemia. Hepatology 1997; 25: 11601165.

[37] Tsuboi Y, Fujino Y, Kobayashi K, Saheki T, Yamada T. High serum pancreatic secretory trypsin inhibitor before onset of type II citrullinemia. Neurology 2001; 57: 933.

[38] Tanaka T, Nagao M, Tsutsumi H. Application of mutation analysis for the previously uncertain cases of adult-onset type II citrullinemia (CTLN2) and their clinical profiles. Tohoku J Exp Med 2002; 198: 89-97.

[39] Hagiwara N, Sekijima Y, Takei Y, Ikeda S, Kawasaki S, Kobayashi K, et al. Hepatocellular carcinoma in a case of adult-onset type II citrullinemia. Intern Med 2003; 42: 978-982.

[40] Tsai CW, Yang CC, Chen HL, Hwu WL, Wu MZ, Liu KL, et al. Homozygous SLC25A13 mutation in a Taiwanese patient with adult-onset citrullinemia complicated with steatosis and hepatocellular carcinoma. J Formos Med Assoc 2006; 105: 852-856.

[41] Tokuhara D, Iijima M, Tamamori A, Ohura T, Takaya J, Maisawa S, et al. Novel diagnostic approach to citrin deficiency: Analysis of citrin protein in lymphocytes. Mol Genet Metab 2007; 90: 30-36.

[42] Moriyama M, Li MX, Kobayashi K, Sinasac DS, Kannan Y, Iijima M, et al. Pyruvate ameliorates the defect in ureogenesis from ammonia in citrin-deficient mice. J Hepatol 2006; 44: 930-938. 


\section{Figure Legends}

Figure 1. Liver histology of CTLN2 patients.

(A) Liver specimen obtained from patient No. 3. Azan-Mallory staining showed the presence of bridging fibrosis with perisinusoidal fibrosis. Macrovesicular steatosis and mild lobular inflammation were observed (Azan-Mallory staining, x 40; hematoxylin and eosin staining, $\mathrm{x} 100$ ).

(B) Liver specimen obtained from patient No. 6. Diffuse macrovesicular steatosis mainly present in zone 3 was observed. Ballooned hepatocytes containing Mallory hyaline were also found (arrows) (Azan-Mallory staining, x 40; hematoxylin and eosin staining, x 100 and x 600).

Figure 2. Comparison of BMI and serum PSTI between fatty liver patients without (-) and with (+) SLC25A13 gene mutations.

Each value was plotted, and median values were indicated in the lines. The cut-off values of BMI and serum PSTI were calculated as $20 \mathrm{~kg} / \mathrm{m}^{2}$ and $29 \mathrm{ng} / \mathrm{mL}$, respectively. $* * *, P<0.001$

Figure 3. A proposed mechanism of steatogenesis in patients with citrin deficiency.

(A) NADH-supplying systems into mitochondria in human hepatocytes. The malate-aspartate shuttle plays a central role in translocating NADH from the cytosol to mitochondria, and citrin (aspartate-glutamate carrier, AGC) is one of the essential components of this pathway. Asp, aspartate; Cit, citrate; CT, citrate transporter; cyt, cytosol; Glu, glutamate; IM, intermembrane space; - KG, - -ketoglutarate; Mal, malate; mit, mitochondrial matrix; NADH, nicotinamide adenine dinucleotide; OAA, oxaloacetate; OMC, oxoglutarate malate transporter.

(B) Changes in NADH-supplying systems caused by citrin deficiency in human hepatocytes. Citrin deficiency causes loss of the malate-aspartate shuttle and compensatory up-regulation of the malate-citrate shuttle, resulting in increased citrate in the cytosol. Abbreviations are identical to those in panel A.

(C) Changes in fatty acid metabolism in patients with citrin deficiency. Overproduction of fatty acids due to increases in cytosolic citrate and enhancement of fatty acid uptake into hepatocytes are proposed to be associated with steatogenesis. ACL, ATP-citrate lyase; CD36; fatty acid translocase; Cit, citrate; FA, fatty acid; TG, triglyceride; cyt, cytosol; mit, mitochondrial matrix. 